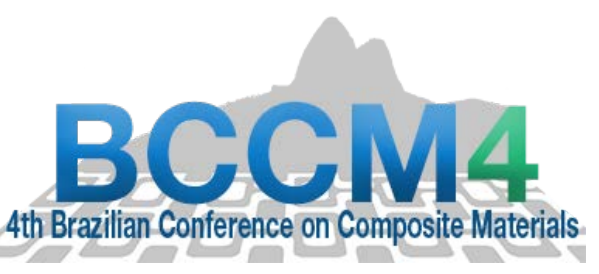

\title{
ARTIFICIAL NEURAL NETWORKS APPLYING TO DETECT DAMAGE IN CARBON FIBER/EPOXY COMPOSITES.
}

\author{
Luísa R. Völtz, Eduardo L. Cardoso and Ricardo De Medeiros. \\ Department of Mechanical Engineering, Santa Catarina State University, Brazil.
}

https://doi.org/10.21452/bccm4.2018.16.02

\begin{abstract}
Artificial Neural Networks (ANNs) have emerged as one of the most useful tools in Artificial Intelligence (AI), being used in the most applications, such as engineering, economics, and health. Due to their great capacity for learning, adaptation, and generalization, ANNs can handle linear and nonlinear models that other methods are not capable to solve. Composite materials are increasingly used in critical and demanding applications, mainly due to its high specific strength and stiffness. However, the challenging of the current Structural Health Monitoring (SHM) methodologies includes identification, detection, and quantification of the damage and, also the prediction of the residual resistance/life of the structure. Therefore, this work proposes a methodology based on ANNs to detect damage in composite structures. Initially, the vibrationbased method was applied using Frequency Response Functions (FRFs) along with Principal Component Analysis (PCA). This tool seeks to reduce the dimensionality of the original data while maintaining its characteristics. Next, a multi-layer neural network was developed for detecting damage in composite plates made of Carbon Fiber Reinforced Polymer (CFRP). Finally, it is discussed the potentialities and limitations of the methodology for use in damage detection systems.
\end{abstract}

Key-words: Artificial Neural Networks (ANNs), composite materials, damage detection, Principal Component Analysis (PCA).

\section{INTRODUCTION}

Composite material, which combines high performance with low weight, are constantly used in many applications, such as the automotive, sporting and aerospace industries, replacing conventional materials due to their excellent mechanical properties, such as high stiffness and corrosion resistance. The advantages of composite materials are many, including fatigue strength and good design practices [1]. However, they have disadvantages due to their micromechanical interactions and their particular failure modes, such as matrix cracking, fiber rupture, interfacial debonding and delamination [2]. Therefore, as any structure, the monitoring of material condition must be performed to ensure its integrity. Many techniques of damage detection use structure vibration responses, including FRFs, natural frequencies and vibration modes [3]. The main idea 
behind damage detection techniques based on dynamic changes is the fact that these variables are all functions of mass, stiffness, and damping and therefore it is reasonable to assume that the existence of damages leads to change of such properties [4]. On the other hand, ANN is a processor made up of processing units that has the natural tendency to store knowledge acquired from its environment through a learning process and makes it available when required for use [5]. After the learning process, the network is then able to generalize the acquired knowledge, making it possible to estimate solutions that were unknown [6]. Brito et al. [7] used ANN to predict the dynamic-mechanical behavior of epoxy resin with carbon fiber composites. The input parameters for the ANN were fiber orientation and response time measured by vibration tests and the output represented the amplitude. Al-Assaf and Kadi [8] predict the fatigue life of glass fiber epoxy composites with a range of fiber orientation using ANN and the network was able to give accurate results when comparing with experimental data.

Although many studies have been carried out using neural networks in damage detection, fatigue studies or prediction of mechanical behavior, few studies are related to composite materials [8]. Therefore, this work aims to present a methodology, based on the use of neural networks and dynamic characteristics of the structure, to be sought for the damage detection in composite plates made of epoxy resin reinforced by carbon fiber.

\section{MATERIALS AND METHODS}

Sixteen composite plates produced from carbon fibers with epoxy resin through the filament winding process were studied. The carbon fiber composites were divided into two groups: eight plates with twelve layers and stacking orientation [0/15/-15/0/15/-15]s and eight plates with eight layers and stacking orientation [0]8. The average thickness for the first group was $3.39 \mathrm{~mm}$ ( standard deviation $=0.07$ ) and for the second $2.23 \mathrm{~mm}$ (standard deviation $=0.02$ ). The experimental procedure was carried out by Medeiros [9] and is shown in Fig 1.

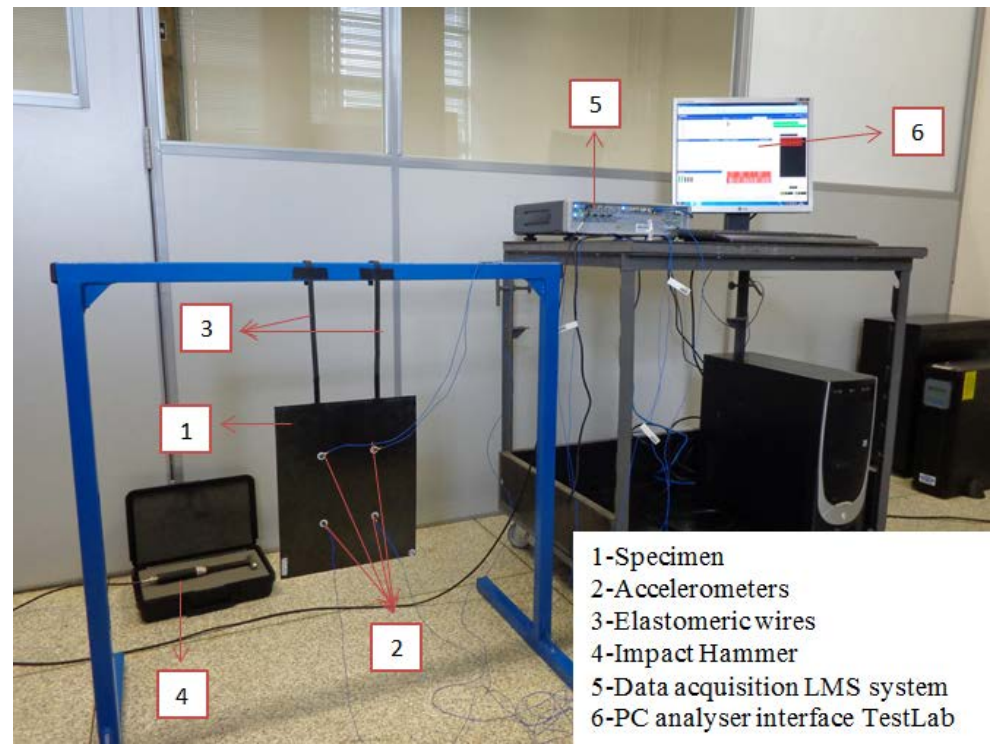

Figure 1: Experimental procedure [9]. 
Initially, the dynamic response of each healthy laminated plate was obtained by using accelerometers in free-free boundary conditions. The impulse input by impact hammer was applied on a fixed point on the back side of the plate. In the vibration tests, the analyzed frequency range was $0-1024 \mathrm{~Hz}$, in a total of 2048 spectral points. Each FRF was obtained through an average of five samples, aiming to reduce the effects of variation [9].

The second stage consists of the damage test phase. For the $[0]_{8}$ orientation group, five plates were damaged by impact loading, one was damaged by drilling a center hole and other two kept undamaged. For the [0/15/-15/0/15/-15]s plates, four were damaged by impact loading, two by delamination and other two kept undamaged. The damaged plates were analyzed again through the use of vibration methods monitored by accelerometers. In total 56 FRFs for [0] 8 orientation plates were obtained, 32 FRFs for healthy cases and 24 FRFs for damaged cases. For [0/15/15/0/15/-15]s plates, a total of 48 FRFs was obtained, 24 for undamaged cases and 24 for damaged cases.

Recently, methods involving ANNs have been considered state-of-the-art to predict a behavior of a system. The intention is to establish a multidimensional and non-linear correlation between the input data and the corresponding output neuron. Each neuron, in each layer, interconnects through synaptic weights, and thus the information crosses from one layer to another until the exit of the network [7]. In this way, a forward neural network was developed to classify patterns of vibration responses of intact and damaged composite plates. Due to a large number of inputs associated with each FRF and the consequently large number of connections required to form a suitable ANN topology, it was studied a methodology to reduce the dimension of the experimental data, called PCA. It is a statistical technique that uses an orthogonal projection, transforming a set of correlated variables into a set of uncorrelated variables, called principal components (PCs). The aim of the PCA is a dimensional reduction and elimination of the noise present in the original data [4].

The ANN was implemented in Julia language [10] using steepest descent with backtracking line search algorithm and automatic differentiation to evaluate the gradients. A moment term was used to accelerate the gradient descent in direction of the error function [5]. For each stack orientation, a new ANN was created to achieve the best result according to each case. After using the PCA, the data were split into three data sets: training, validation, and testing. The training set was used as input into the neural network, and an error calculation was performed by using feedforward propagation and square-root or cross-entropy functions. The validation set was then used to monitor the behavior of the network simultaneously with the training set during the learning phase, to monitor the best values of the hyperparameters and topology. After the learning phase, the network was then tested using the last set of data, that it wasn't used during training.

The confusion matrix is a very useful technique for evaluating the errors distributed across the classes. Tab. 1 shows an example of the confusion matrix. TP means true positive that is the samples of the damaged class were correctly classified as damaged. FP means false positive, is the number of samples not belonging to damaged class but misclassified into damaged class. FN, false negative, is the number of samples of damaged class misclassified as an undamaged class. TN, true negative, is the number of samples of undamaged class that were classified correctly. The accuracy can be calculated as the trace of the matrix divided by the total number of the samples [11]. Recall (R), or sensitivity, is the proportion of positive cases that were correctly identified, defined as

$R=T P /(T P+F N)$ 
Precision $(\mathrm{P})$ is the proportion of the predicted positive cases that were correct, defined as $P=T P /(T P+F P)$.

Table 1: Confusion Matrix.

\begin{tabular}{|c|c|c|c|}
\cline { 3 - 4 } \multicolumn{2}{c|}{} & \multicolumn{2}{|c|}{ Actual class } \\
\cline { 3 - 4 } \multicolumn{2}{c|}{} & Yes & No \\
\hline \multirow{2}{*}{ Predict class } & Yes & TP & FP \\
\cline { 2 - 4 } & No & FN & TN \\
\hline
\end{tabular}

\section{RESULTS AND DISCUSSION}

Each stacking orientation group was studied separately, due to the large differences in dynamic behavior among them.

\subsection{Composite plates with stacking orientation [0/15/-15/0/15/-15]s}

Figure 2 shows the experimental real, imaginary and magnitude graphs for one plate with stacking orientation [0/15/-15/0/15/-15]s, considering undamaged and damaged cases. It is possible to observe that the differences between undamaged and damaged are very subtle. In addition, comparing the damage and undamaged FRFs, the differences in amplitude are more perceptible in both the imaginary part and magnitude.
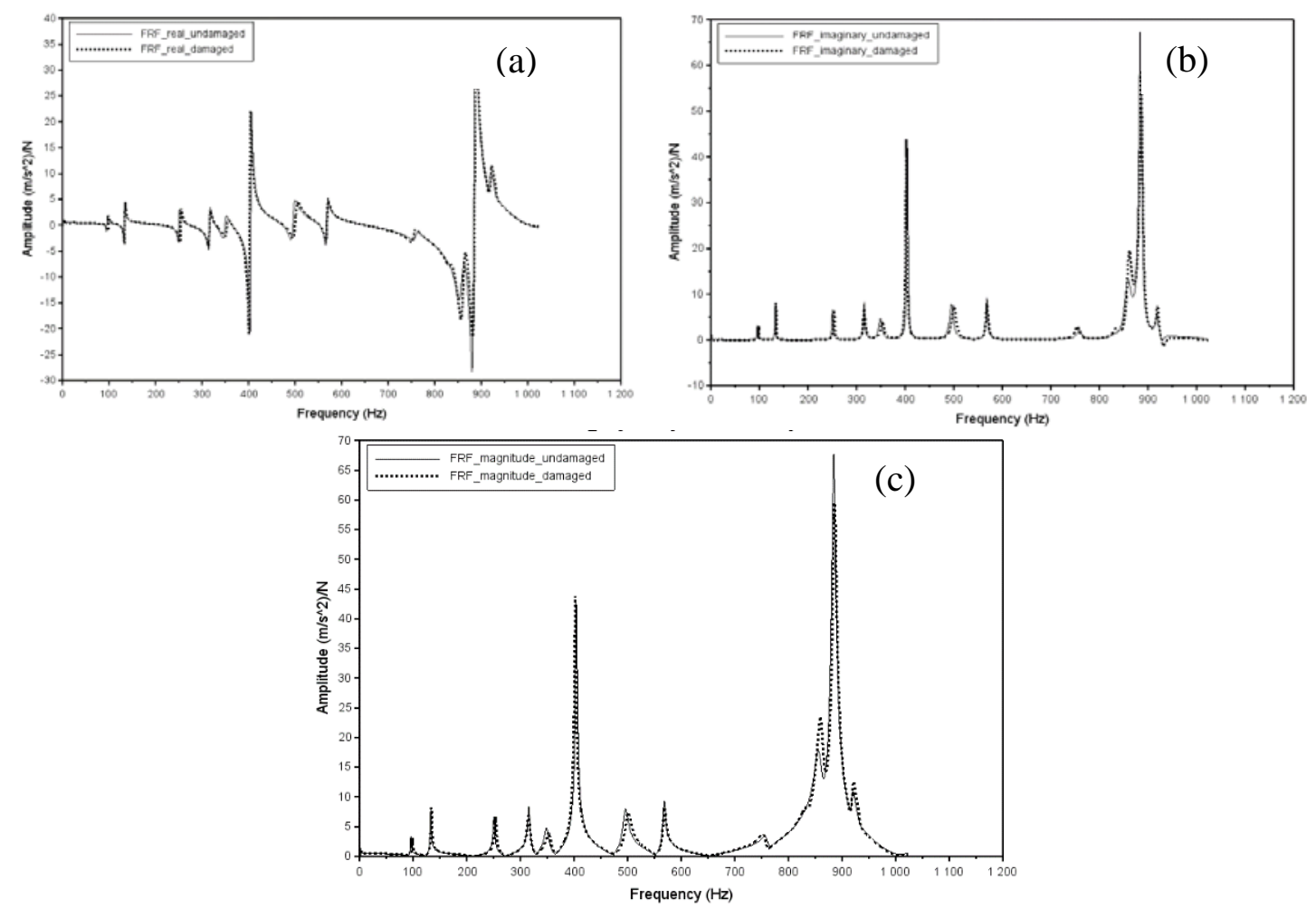

Figure 2: (a) real part, (b) imaginary part and (c) magnitude FRFs.

The data dimensionality was reduced using the PCA, and for the first 30 PCs, the total variance was $98.85 \%$, using only real values of the FRFs. The data were split into $62 \%$ for the training set, $21 \%$ for the validation set and $17 \%$ for the testing set. The resulting 30 variables were introduced 
as input in the ANN. Different topologies and values of parameters were taken. The best topology was 30 inputs, 20 neurons in the first hidden layer, 10 neurons in the second hidden layer and 1 output ( 0 for healthy and 1 for damaged conditions). The moment term used was 0.1 . The learning rate was kept fixed with 0.1 . The logistic function as activation function was applied. The crossentropy cost function was used and in 400 iterations the network reached $100 \%$ of accuracy in the training set, $80 \%$ in the validation set and $87.7 \%$ in the testing set. Table 2 shows the confusion matrix for the testing set with $87.5 \%$ of accuracy, $100 \%$ for precision and $75 \%$ for recall.

Table 2: Confusion matrix for [0/15/-15/0/15/-15]s testing set, considering the real part.

\begin{tabular}{|c|c|c|c|c|}
\cline { 3 - 5 } \multicolumn{2}{c|}{} & \multicolumn{2}{c|}{ Actual Class } & \multicolumn{1}{c}{} \\
\cline { 3 - 5 } \multicolumn{2}{c|}{} & Damaged & Undamaged & TOTAL \\
\hline \multirow{2}{*}{ Predict Class } & Damaged & $\mathrm{TP}=3$ & $\mathrm{FP}=0$ & 3 \\
\cline { 2 - 5 } & Undamaged & $\mathrm{FN}=1$ & $\mathrm{TN}=4$ & 5 \\
\hline & TOTAL & 4 & 4 & $\mathbf{8}$ \\
\hline
\end{tabular}

After performing the first analysis, it was observed that the influence of the imaginary part in the $[0 / 15 /-15 / 0 / 15 /-15]_{\text {s }}$ stacking sequence could be important. A new simulation using magnitude values of FRFs was then studied. After pre-processing using PCA, new 20 PCs was calculated with $98.10 \%$ of the total variance. The resulting 20 variables were introduced as input in the new ANN. After some simulations, the best result was found. The topology was 20 inputs, 12 neurons in the hidden layer and 1 output. The value of 0.7 for the moment term was used and backtracking line search. The logistic function and the $\mathrm{L}_{2}$ cost function were applied. After 150 iterations the network reached $100 \%$ of accuracy in the training set, $90 \%$ in the validation set and $100 \%$ in the testing set, showing good generalization. The best ANN results were obtained by using only magnitude values. Figure 3 shows the ANN errors and accuracy curves during the training and the validation phases. The continuum lines are error curves, and the dot lines are the accuracy curves for [20/12/1] topology, the final error for the training phase was 0.0009 .

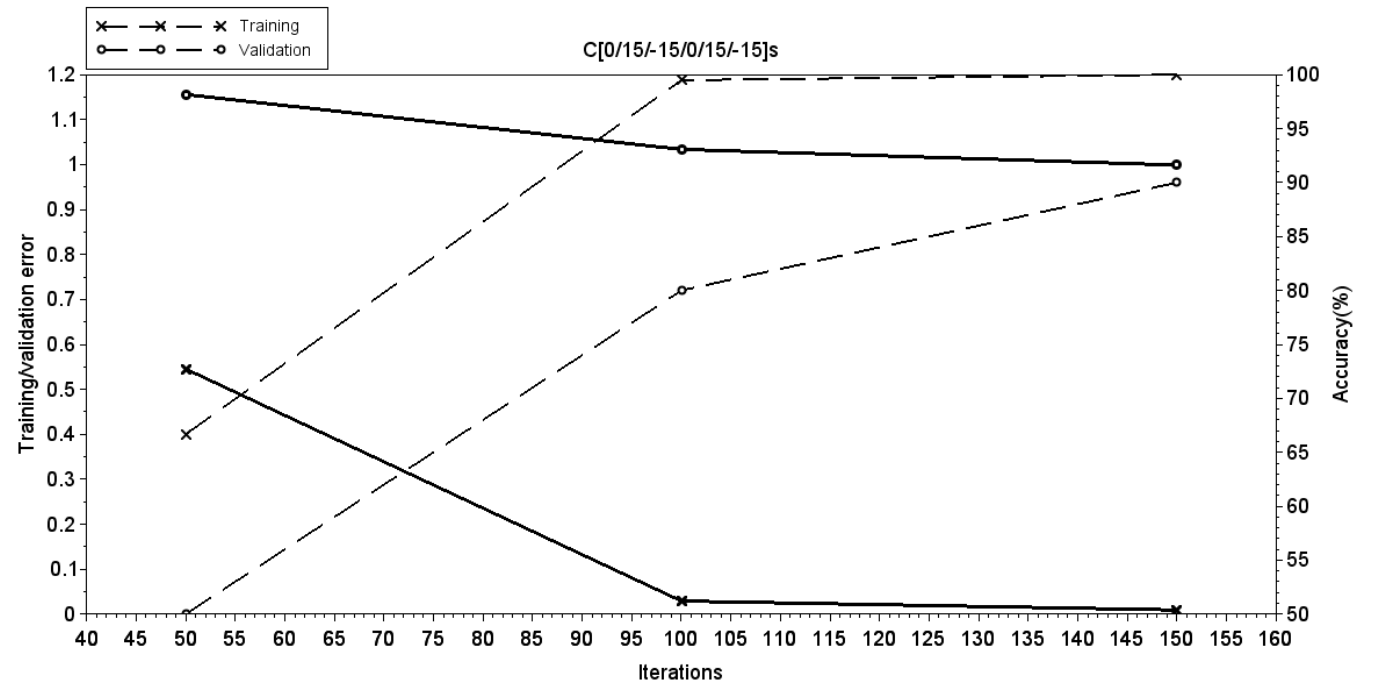

Figure 3:

ANN

Error and

Accuracy

for

$[0 / 15 /-$

$15 / 0 / 15 /-$

$15]_{S}$

plates.

3.2

\section{Composite plates with orientation $[0]_{8}$}

Figure 4 shows the experimental real, imaginary and magnitude graphs for one plate with stacking orientation of $[0]_{8}$, considering undamaged and damaged cases. The differences between 
undamaged and damaged are very clear, with changes in the natural frequencies, amplitude, and phases, especially on imaginary part.
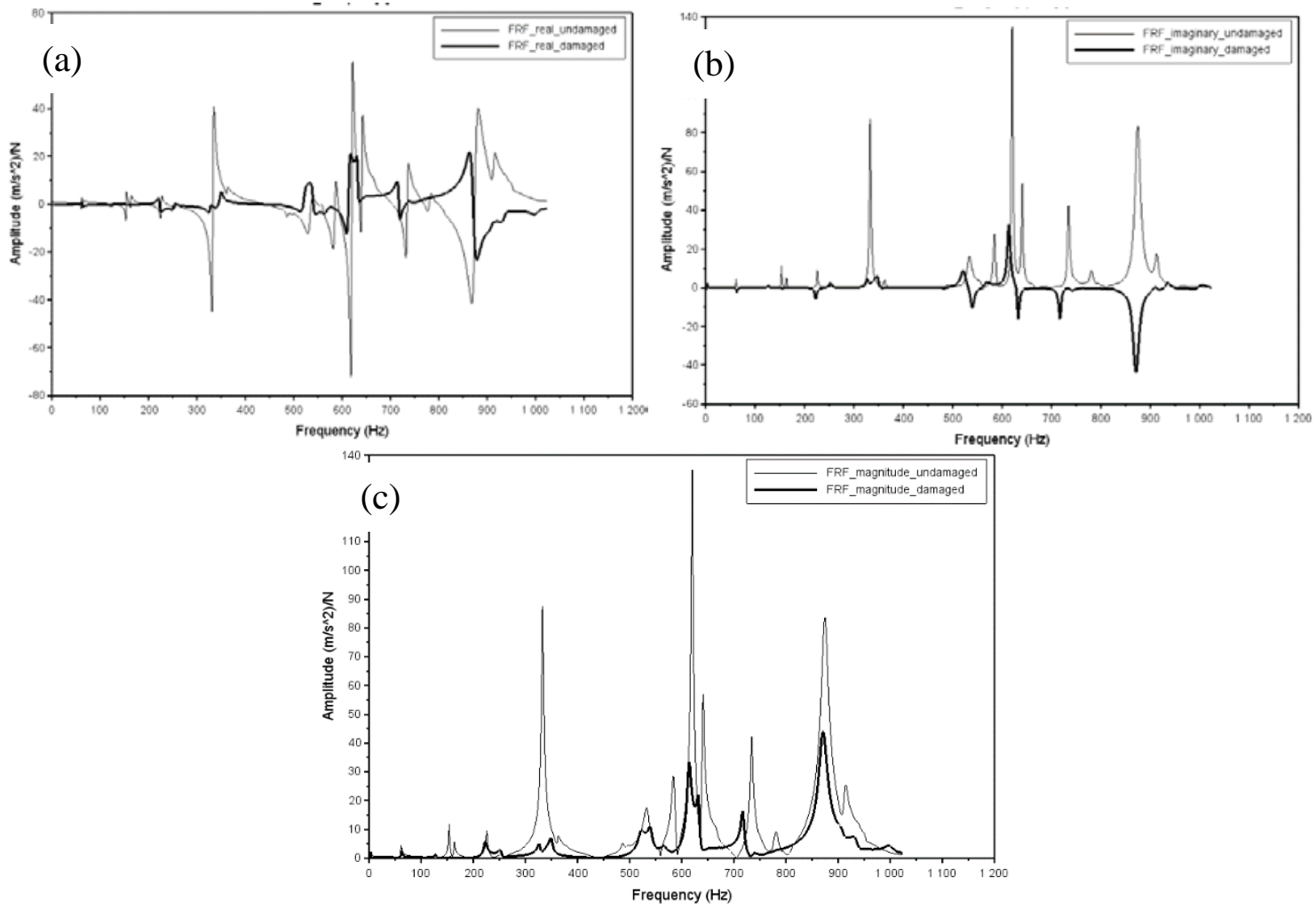

Figure 4: (a) real part, (b) imaginary part and (c) magnitude FRFs.

After dimension reduction, the first 20 PCs were evaluated with $98.55 \%$ of total variance using only real values of the FRFs. The data were split into $61 \%$ for the training set, $21 \%$ for the validation set and $18 \%$ for the testing set. The resulting 20 variables were introduced as input and different topologies, and values of parameters were considered until reaching the best solution. The best topology was 20 inputs, 6 neurons in the hidden layer and 1 output ( 0 for healthy and 1 for damaged conditions). The moment term used was 0.7 and the backtracking line search. Logistic and $L_{2}$ cost functions were applied, and in just 150 iterations the network reached $100 \%$ of accuracy in training, validation and testing sets.

To evaluate the influence of the imaginary part in the $0^{\circ}$ orientation the same procedure was done. The magnitude values were calculated and passed through PCA, and the first 20 PCs were taken with 99.19\% of total variance. The best result was achieved with 20 inputs, 9 neurons in the first hidden layer, 2 neurons in the second hidden layer and 1 output. The moment term was 0.7 , backtracking line search, and the logistics and the $\mathrm{L}_{2}$ cost functions were used. After 300 iterations the network reached $100 \%$ of accuracy in the training set, $83.3 \%$ in the validation set and $90 \%$ in the testing set. Table 3 shows the confusion matrix for the testing set, with $90 \%$ for accuracy, $66.7 \%$ for recall and $100 \%$ for precision.

After comparing the results using real and magnitude values, the best ANN result was obtained by using only real part of the FRF for stacking orientation $[0]_{8}$. Figure 5 shows the curves between errors and accuracy during the training and the validation phases. The continuum lines are error curves, and the dot lines are the efficiency curves for [20/6/1] topology. The final error for training was 0.009 and $100 \%$ of accuracy for both sets. 
Table 3: Confusion matrix for $[0]_{8}$ testing set, considering magnitudes values.

\begin{tabular}{|c|c|c|c|c|}
\cline { 3 - 5 } \multicolumn{2}{c|}{} & \multicolumn{2}{c|}{ Actual Class } & \multicolumn{1}{|c|}{} \\
\cline { 3 - 5 } \multicolumn{2}{c|}{} & Damaged & Undamaged & TOTAL \\
\hline \multirow{2}{*}{ Predict Class } & Damaged & $\mathrm{TP}=2$ & $\mathrm{FP}=0$ & 2 \\
\cline { 2 - 5 } & Undamaged & $\mathrm{FN}=1$ & $\mathrm{TN}=7$ & 8 \\
\hline & TOTAL & 3 & 7 & $\mathbf{1 0}$ \\
\hline
\end{tabular}

Finally, as observed, all the ANNs showed good learning and generalization. The best result for each orientation agreed to what was observed in the originals FRFs. Large differences can be observed in real, imaginary and magnitude values for [0] 8 stacking orientation. During ANN simulations it was verified that when using only real values as input the behavior of the network was better than when using magnitude values. It was probably due to the fact that in the real part of the FRF there are inversions of the phases in almost all the modes between intact and damaged plates. This situation was hidden when working with magnitude values, as seen in Fig. 4(c). The inversion of the phase, differentiating the intact from the damaged ones, can contribute to feature extraction during the learning and then to the classification performed by the network. The network accuracy using real values was $100 \%$, for all the three sets, and for testing set the ANN precision and recall were $100 \%$ either. It means that ANN can correctly classify all the classes studied.

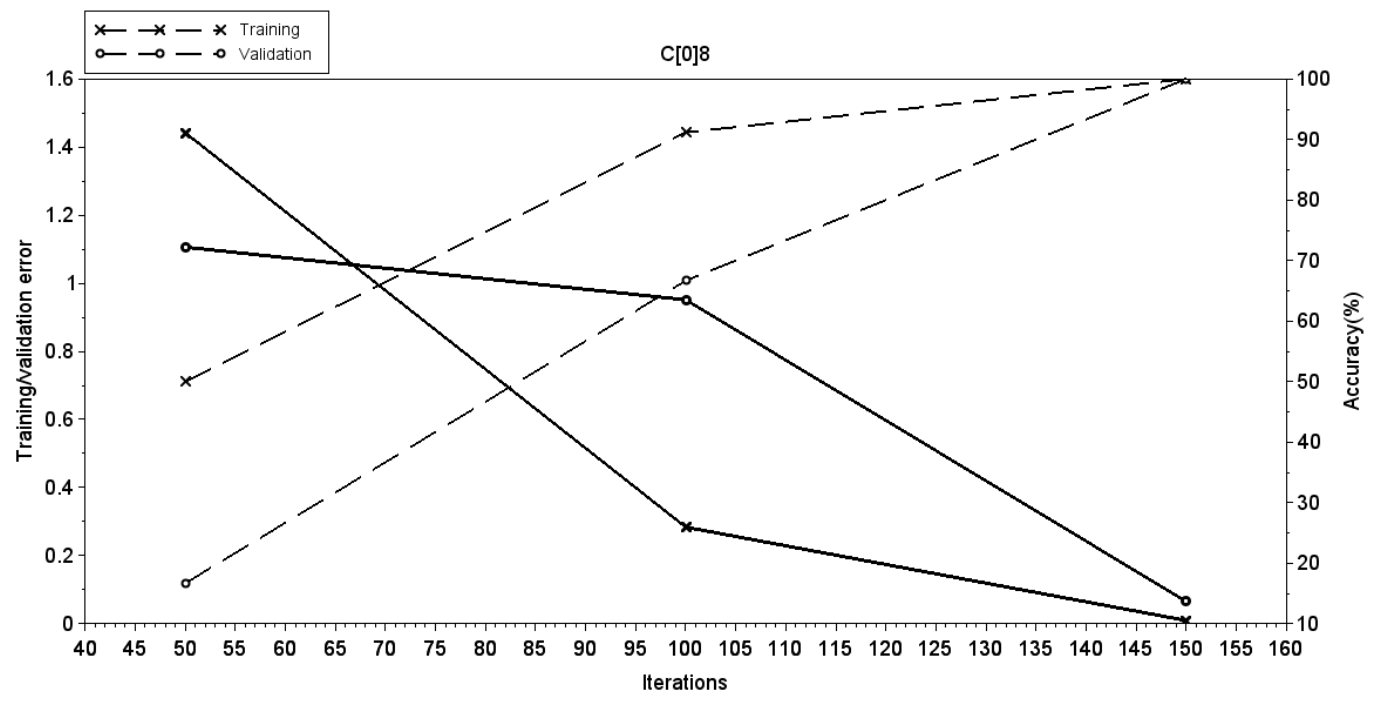

Figure 5: ANN Error and Accuracy for $[0]_{8}$ plates.

For $[0 / 15 /-15 / 0 / 15 /-15]_{S}$ laminates the best result was obtained when the magnitude values were used. This is probably due to the fact that values in magnitude carry more information about each integrity condition. As the FRFs are similar, the features acquired only by the real part created a more complex decision boundary between healthy and unhealthy cases. Adding the features acquired by the imaginary part, the curves changed differentiating the dynamic behavior between them. Due to this feature addition, the network began to rank better and evaluate the presence or not of the damage and thus make its decision correctly. The network accuracy was $100 \%$ for the training, $90 \%$ for the validation set and $100 \%$ for the testing set, showing very good precision (100\%), which means the network didn’t classify any samples that shouldn't be classified. 
$4^{\text {th }}$ Brazilian Conference on Composite Materials. Rio de Janeiro, July $22^{\text {nd }}-25^{\text {th }}, 2018$

\section{CONCLUSIONS}

In this work, ANNs combined with vibration-methods and PCA were used to detect damages in epoxy/carbon reinforced composites. Several architectures and hyperparameters values were evaluated to find the best solution to classify each integrity class. A confusion matrix was used to evaluate the accuracy, recall, and precision of the ANN and its effectiveness for pattern recognition. The best result showed that for real values of the FRF the ANN performed better with $[0]_{8}$ orientation, with $100 \%$ of accuracy in the training, the validation, and the testing sets. Opposite, laminates plates with orientation [0/15/-15/0/15/-15]s the ANN generalized better when using magnitude values of the FRFs, with an accuracy of $100 \%$ for the training and the testing sets, showing good generalization and $90 \%$ for the validation set. Therefore, this methodology can help in the development of SHM systems and, consequently, in the damage detection.

\section{ACKNOWLEDGMENTS}

The authors acknowledge the financial support of the State Research Founding Agency (FAPESC process number: 2017TR1747 and 2017TR784). As well as, Sao Paulo State Research Foundation (FAPESP process number: 2012/01047-8 and 2015/15221-8), Coordination for the Improvement of the Higher Level Personnel (CAPES process number: 011214/2013-09), National Council for Scientific and Technological Development (CNPq process number: 401170/2014-4 and 310094/2015-1). The authors also would like to thank Navy Technological Centre (CTM - Brazil) for manufacturing specimens.

\section{REFERENCES}

[1] Campbell, F.C. 'Structural composite materials'. ASM International. (Ohio, EUA, 2010).

[2] Montalvão, D., Maia, N.M.M. and Ribeiro, A.M.R. 'A review on vibration-based structural health monitoring with special emphasis on composite materials'. Shock and Vibration Digest, 38 (4) (2006) 295 - 324.

[3] Bandara, R.P., Chan, T.HT. and Thambiratnam, D. P. 'Structural damage detection method using frequency response functions'. Structural Health Monitoring. 13 (4) (2014). 418 - 429

[4] Li, J., Dackermann, U., Xu, Y.L. and Samali, B. 'Damage identification in civil engineering structures utilizing PCA-compressed residual frequency response functions and neural networks ensembles'. Structural Control Health Monitoring. 18 (2011) 207-226.

[5] Haykin, S. 'Neural networks: a comprehensive foundation'. Translate by Paulo Engel. 2nd Edn. (Bookman, Porto Alegre, 2001).

[6] Silva, I.N, Spatti, D.H. and Flauzino, R. 'Artificial neural networks'. 2nd Edn. (Artliber, São Paulo, 2016).

[7] Brito, Jr et al. 'Redes Neurais Artificiais aplicadas para a predição do comportamento dinâmicomecanico de compositos de matriz epóxi reforçados com fibras de carbono’. Revista Matéria 12 (1) (2007) 346-357.

[8] Al-Assaf, Y. and Kadi, El. 'Fatigue life prediction of unidirectional glass fiber/epoxy composite laminae using neural networks’. Composite Structures 53 (2001) 65-71.

[9] Medeiros, R. 'Development of a criterion for predicting residual strength of composite structures damaged by impact loading’. São Carlos University (2016).

[10] Bezanson, J., Edelman, A., Karpinski, S. and Shah, V.B. 'Julia: A fresh approach to numerical computing’. CoRR, abs/1411.1607 (2014). Disponível em: <http://arxiv.org/abs/1411.1607>. Acess: march 15th, 2018. 
$4^{\text {th }}$ Brazilian Conference on Composite Materials. Rio de Janeiro, July $22^{\text {nd }}-25^{\text {th }}, 2018$

[11] Kuncheva, L. 'Combining Pattern Classifiers: Methods and Algorithms'. (John Wiley \& Sons, New Jersey, 2004). 